\title{
Beamforming matrix quantization with variable feedback rate
}

\author{
Chau Yuen ${ }^{1 *}$, Sumei Sun ${ }^{2}$, Mel Meau Shin $\mathrm{Ho}^{3}$ and Zhaoyang Zhang ${ }^{4}$
}

\begin{abstract}
We propose a new technique to quantize and feedback the parameters when a beamforming matrix is compressed with the Givens Rotation (GR). We suggest to feedback the parameters with variable feedback rate, and use efficient source coding and codebook to quantize the GR parameters. The variable feedback rate means that the number of bits used to represent the quantized beamforming matrix is based on the value of the matrix itself. And due to the non-uniform distribution of the GR parameters, source coding and code book can be designed to quantize those parameters in a more effective manner. Compared with the fixed feedback rate scheme, the proposed method delivers a better performance without incurring additional feedback bandwidth.
\end{abstract}

\section{Introduction}

Multiple transmit and receive antennas system has been adopted in several communication standards in order to achieve a higher throughput. The open-loop multipleinput multiple-output (MIMO) technique has already been shown to achieve a high performance gain. With the availability of either the full or partial channel state information (CSI) at the transmitter, we can achieve further performance gain or receiver complexity reduction. Such closed-loop schemes have been considered in many communication standards for application of beamforming or multi-user precoding.

However, CSI estimation for the downlink channel at the base station is not possible in Frequency Division Duplex systems. It is also not straightforward to implement CSI estimation in Time Division Duplex (TDD) systems due to the mismatch in the radio front end. Hence in general, the CSI will be estimated at the mobile clients and be sent back to the base station. For example, in the 802.11n wireless LAN system, when the system is operated in TDD mode, the channel can either be estimated by the transmitter through calibration or the channel is fed back by the receiver [1]. This unfortunately requires a high and undesirable feedback bandwidth.

Another popular way to reduce the amount of CSI feedback is through differential encoding [2,3]. However, such

\footnotetext{
* Correspondence: Email: yuenchau@sutd.edu.sg

${ }^{1}$ Singapore University of Technology and Design, Singapore, Singapore Full list of author information is available at the end of the article
}

technique suffers accumulated error propagation. Therefore, the mobile client often computes the beamforming matrix, which is usually a unitary matrix and "compress" such a matrix before feeding back to the base station. The "compression" can significantly reduce the feedback bandwidth requirement. And 802.11 ac wireless LAN system [4] adopts this methodology to feedback the beamforming matrix for single- and multi-user MIMO. Although we only use unitary beamforming matrix as an example in this article, the techniques that are discussed in this article apply to the feedback of channel matrix as well. For example, we may perform singular value decomposition (SVD) on the channel matrix, and feedback the eigenvalues and both the left and right eigenvectors matrices, which are both unitary.

There have been several proposals in the literature to compress the beamforming vector. One is codebook based such as the vector quantization (VQ) scheme proposed in [5-10], and another is by using the Givens Rotation (GR) [1,4,11-13]. Compared with the GR-based scheme, the VQ approach requires a higher storage, as a set of codebooks is needed for a particular antenna setting. It has a higher complexity than the GR approach, especially when the number of codewords in the codebook increases. Due to these reasons, the GR approach has been adopted in the $802.11 \mathrm{n}$ and 802.11 ac standards $[1,4]$.

In this article, we investigate an effective approach to quantize and feedback the GR parameters that compress the beamforming matrix. The proposed scheme is capable of achieving a better performance, in the absence of

\section{倠}

(c) 2012 Yuen et al.; licensee Springer. This is an Open Access article distributed under the terms of the Creative Commons Attribution License (http://creativecommons.org/licenses/by/2.0), which permits unrestricted use, distribution, and reproduction in any medium, provided the original work is properly cited. 
extra bandwidth, than existing techniques that quantize and feedback the GR parameters.

\section{Signal model MIMO model}

Consider a point-to-point MIMO channel with $N_{T}$ transmit antennas and $N_{R}$ receive antennas, the $N_{T} \times 1$ transmitted signal is denoted by $\mathbf{x}$ and the $N_{R} \times N_{T}$ channel denoted by $\mathbf{H}$. The $N_{R} \times 1$ received signal y can be expressed as

$$
\mathbf{y}=\mathbf{H x}+\mathbf{n}
$$

To demonstrate the idea of beamforming, we use the eigen-subspace beamforming as an example. By using SVD, a MIMO channel $\mathbf{H}$ can be decomposed into

$$
\mathbf{H}=\mathbf{U D V}^{\mathrm{H}}
$$

where $\mathbf{U}$ of size $N_{R} \times R$ and $\mathbf{V}$ of size $N_{T} \times R$ are both unitary matrices, and $\mathbf{D}$ is an $R \times R$ diagonal matrix consisting of the singular values of $\mathbf{H}$ as its diagonal elements, and $R$ is the rank of $\mathbf{H}$. In order to perform eigen-subspace beamforming, $\mathbf{V}$ needs to be fed back to the base station. An effort to reduce the amount of information in $\mathbf{V}$ was reported in [11-13] where a matrix $\boldsymbol{\Sigma}$ was multiplied with $\mathbf{V}$ to form $\overline{\mathbf{V}}$, such that the last row of $\overline{\mathbf{V}}$ consists of only real numbers. Hence, we may re-express (2) as:

$$
\mathbf{H}=\mathbf{U D} \boldsymbol{\Sigma} \overline{\mathbf{V}}^{\mathrm{H}}=\mathbf{U} \overline{\mathbf{D}} \overline{\mathbf{V}}^{\mathrm{H}} \text { where } \overline{\mathbf{D}}=\mathbf{D} \boldsymbol{\Sigma}
$$

where

$$
\boldsymbol{\Sigma}=\operatorname{diag}\left[\exp \left\{j * \arg \left(\mathbf{v}_{N_{T}}^{\mathrm{H}}\right)\right\}\right]
$$

and $\mathbf{v}_{N_{T}}^{\mathrm{H}}$ represents the last column of $\overline{\mathbf{V}}^{\mathrm{H}}$.

$$
\mathbf{y}=\mathbf{H} \mathbf{x}+\mathbf{n}=\left(\mathbf{U} \overline{\mathbf{D}} \overline{\mathbf{V}}^{\mathrm{H}}\right) \mathbf{x}+\mathbf{n}
$$

To transmit data in the first $K$ eigen modes (where $K \leq$ $R$ ), the beamforming matrix $\mathbf{W}$ is simply the first $K$ column vectors of $\overline{\mathbf{V}}$ :

$$
\mathbf{W}=\overline{\mathbf{V}}_{(1: K)}
$$

The transmitted signal is related to the $K \times 1$ data signal $\mathbf{u}$ by

$$
\mathbf{x}=\mathbf{W} \mathbf{u}
$$

In order to retrieve $\mathbf{u}$, the mobile client multiplies the received signal with $\mathbf{U}^{\mathrm{H}}$,

$$
\mathbf{a}=\mathbf{U}^{\mathrm{H}} \mathbf{y}=\overline{\mathbf{D}}_{(1: K)} \mathbf{u}+\tilde{\mathbf{n}}
$$

where $\tilde{\mathbf{n}}$ has the same statistics as $\mathbf{n}$ (as $\mathbf{U}$ is a unitary matrix). Since $\overline{\mathbf{D}}$ is a diagonal matrix, eigen- beamforming leads to simple decoding, as the MIMO channel can be treated as a number of parallel subchannels.

In practice, due to the limited bandwidth in the feedback channel, $\mathbf{W}$ has to be quantized, and the base station receives the quantized version of $\mathbf{W}$, denoted by $\widetilde{\mathbf{W}}$ . We assume that the channels are estimated accurately, and there is no error or delay in the feedback channel. With these assumptions we consider only the impact of quantization error due to limited feedback bandwidth. Hence, $\widetilde{\mathbf{W}}$, instead of $\mathbf{W}$, will be used as the beamforming matrix. In this article, we propose an effective method to quantize $\mathbf{W}$ and it will be shown that we can achieve a better performance than that of existing methods using the same average number of feedback bits.

\section{GR model}

Before we illustrate how the new proposed approach can easily be applied to the GR, we give a brief review on the GR. A unitary matrix, such as $\mathbf{W}$ in our case, can be represented as follows:

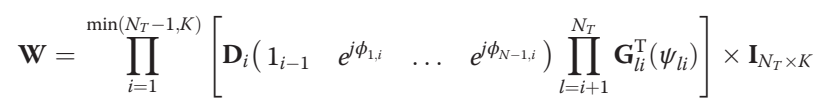

where $\mathbf{D}_{\boldsymbol{i}}$ is a diagonal matrix and $\mathbf{G}$ is defined as

$$
\mathbf{G}_{l i}\left(\psi_{l i}\right)=\left[\begin{array}{ccccc}
\mathbf{I}_{i-1} & \mathbf{0} & \mathbf{0} & \mathbf{0} & \mathbf{0} \\
\mathbf{0} & \cos \left(\psi_{l i}\right) & \mathbf{0} & \sin \left(\psi_{l i}\right) & \mathbf{0} \\
\mathbf{0} & \mathbf{0} & \mathbf{I}_{l-i-1} & \mathbf{0} & \mathbf{0} \\
\mathbf{0} & -\sin \left(\psi_{l i}\right) & \mathbf{0} & \cos \left(\psi_{l i}\right) & \mathbf{0} \\
\mathbf{0} & \mathbf{0} & \mathbf{0} & \mathbf{0} & \mathbf{I}_{N_{T}-1}
\end{array}\right]
$$

Take a $3 \times 2$ unitary matrix $\mathbf{W}$ as example, it can be described as

$$
\begin{aligned}
\mathbf{W} & =\left[\begin{array}{ccc}
e^{j \phi_{11}} & 0 & 0 \\
0 & e^{j \phi_{21}} & 0 \\
0 & 0 & 1
\end{array}\right] \times \mathbf{G}_{21}^{\mathrm{T}}\left(\psi_{21}\right) \mathbf{G}_{31}^{\mathrm{T}}\left(\psi_{31}\right) \\
& \times\left[\begin{array}{ccc}
1 & 0 & 0 \\
0 & e^{j \phi_{22}} & 0 \\
0 & 0 & 1
\end{array}\right] \times \mathbf{G}_{32}^{\mathrm{T}}\left(\psi_{32}\right) \times\left[\begin{array}{ll}
1 & 0 \\
0 & 1 \\
0 & 0
\end{array}\right]
\end{aligned}
$$

Hence, the $3 \times 2$ unitary matrices $\mathbf{W}$ can fully be described by just six parameters: $\phi_{11}, \phi_{21}, \psi_{21}, \psi_{31}, \phi_{22}$, and $\psi_{32}$. A $3 \times 1$ unit-norm vector only needs four parameters, namely $\phi_{11}, \phi_{21}, \psi_{21}$, and $\psi_{31}$. Whereas for $2 \times 1$ and $2 \times 2$ cases, two parameters, $\phi_{11}$ and $\psi_{21}$, will be sufficient. The full details can be founded in $[1,4]$.

There are four combinations of bits assigned to the GR parameters in the IEEE 802.11n draft. They can be summarized as follows in the format of $\left(b_{\psi}, b_{\phi}\right)$, namely $(1,3),(2,4),(3,5)$, and $(4,6)$, where $b_{\psi}$ represents the number of bits assigned to $\psi$, and $b_{\phi}$ represents the number of bits assigned to $\phi$. Parameter $\psi$ has a range 
from 0 to $\pi / 2$ whereas $\phi$ spans over a range from 0 to $2 \pi[1,4]$.

Using the above bit assignment, $\psi$ and $\phi$ can be quantized according to (12) (where $\widetilde{\psi}$ and $\widetilde{\phi}$ represent the quantized version of $\psi$ and $\phi$, respectively).

$$
\begin{aligned}
& \widetilde{\psi}=\frac{k \pi}{2^{b_{\psi}+1}}+\frac{\pi}{2^{b_{\psi}+2}} \text { where } k=0,1, \ldots, 2^{b_{\psi}}-1 \\
& \widetilde{\phi}=\frac{k \pi}{2^{b_{\phi}-1}}+\frac{\pi}{2^{b_{\phi}}} \text { where } k=0,1, \ldots, 2^{b_{\phi}}-1
\end{aligned}
$$

The beamforming matrix $\widetilde{\mathbf{W}}$ can be recovered at the base station by using (14):

$$
\widetilde{\mathbf{W}}=\prod_{i=1}^{\min \left(N_{T}-1, K\right)}\left[\mathbf{D}_{i}\left(\begin{array}{llll}
1_{i-1} & e^{j \widetilde{\phi}_{1, i}} & \cdots & e^{j \widetilde{\phi}_{N_{T}-1, i}}
\end{array}\right) \prod_{l=i+1}^{N_{T}} \mathbf{G}_{l i}^{\mathrm{T}}\left(\widetilde{\psi}_{l i}\right)\right] \times \mathbf{I}_{N_{T} \times K}
$$

\section{Methods}

The three basic ideas of the proposed scheme are as follows:

\section{A. Dynamic bit assignment:}

The bits assigned to the GR parameter $\phi$ can be made dependent on the value of $\psi$. When the resolution is "sparse" (which can be predetermined based on the value of $\psi$ ), we use more bits for the quantization of $\phi$; when the resolution is "crowded", we use fewer bits for the quantization of $\phi$. In other words, the bit assignment to $\phi$ is adaptively adjusted based on the value of $\psi$.

\section{B. Efficient source coding:}

Due to the non-uniform distribution of the GR parameters $\psi$, efficient source coding such as the Huffman code [14] can be used to efficiently encode the GR parameter $\psi$ and hence reducing the number of feedback bits required.

\section{Codebook design:}

Due to the same reason of non-uniform distribution, instead of quantizing the GR parameter $\psi$ in a uniform manner, codebook can be designed so as to quantize the parameter in a more effective manner.

Depending on the receiver structure or the design criteria, we can apply each of these ideas separately or jointly. We will illustrate each of the above in more details.

\section{Dynamic bit assignment}

To illustrate the idea of dynamic bit assignment, it is best to make use of a simple example of $2 \times 1$ beamforming vector. Consider a $2 \times 1$ unit-norm vector $\mathbf{w}$ as shown in (15), due to the unit-norm property, it must satisfy the constraints in (16). In addition, there is a matching between the $G R$ view point and the Geometry view point, i.e., both $r_{1}$ and $r_{2}$ are related to $\psi_{21}$ by $r_{1}=$ $\cos \left(\psi_{21}\right)$ and $r_{2}=\sin \left(\psi_{21}\right)$.

$$
\begin{gathered}
\mathbf{w}=\left[\begin{array}{l}
w_{1} \\
w_{2}
\end{array}\right] \\
=\left[\begin{array}{c}
r_{1} e^{j \phi_{11}} \\
r_{2}
\end{array}\right] \quad \leftarrow \text { Geometry view point } \\
=\left[\begin{array}{c}
\cos \psi_{21} e^{j \phi_{11}} \\
\sin \psi_{21}
\end{array}\right] \leftarrow \text { Givens Rotation view point }
\end{gathered}
$$

Since $\mathbf{w}$ is a unit-norm vector, it must satisfy:

$$
\begin{aligned}
& w_{1}^{2}+w_{2}^{2}=1 \\
& \Rightarrow r_{1}^{2}+r_{2}^{2}=1
\end{aligned}
$$

Based on the geometry view point in (15) and the constraints in (16), if we want to reduce the quantization error between the $\widetilde{\mathbf{w}}$ (quantized $\mathbf{w}$ ) with $\mathbf{w}$, we obtain the following insights:

- When $r_{2}$ is large ( $\psi_{21}$ is large), $r_{1}$ will be small; $\phi_{11}$ can have a lower resolution.

- When $r_{2}$ is small ( $\psi_{21}$ is small), $r_{1}$ will be large; $\phi_{11}$ will need a higher resolution.

Hence, the number of bit assigned to $\phi_{11}$ should be a function of $r_{1}$ and $r_{2}$, which is in turn related to the value of $\psi_{21}$ when GR is in use. This observation can be further illustrated in Figures 1 and 2. As shown in Figure 1 , the radii of the two circles represent two possible values of $r_{1}$. This is equivalent to one bit assignment to $\psi_{21}$. When $r_{2}$ is small, $r_{1}$ will be large, as shown by the blue circle marked with " $x$ " at the bottom of Figure 1 . When $r_{2}$ is large, $r_{1}$ will be small, noting the red circle marked with "O" at the top of Figure 1. It can be seen that in this case, if we assign the same number of bits (e. g., 3 bits) to $\phi_{11}$, it corresponds to eight points on each circle. The points on the upper circle are closer to each other, while the points on the lower circle are further apart. In this case, the total number of bits to represent $\mathbf{w}$ is $1+3=4$ bits. And this is the standard way of quantizing $\mathbf{w}$, e.g., in the $802.11 \mathrm{n}$ standard.

To achieve a lower quantization error, we can assign different number of bits to $\phi_{11}$ according to the value of $r_{1}$. For example, as shown in Figure 2, we assign two bits to $\phi_{11}$ when $r_{1}$ is small (i.e., $\psi_{21}$ is large, the upper circle), and we assign 4 bits to $\phi_{11}$ when $r_{1}$ is large (i.e., $\psi_{21}$ is small, the lower circle). It can be seen that the distance between the points are more evenly distributed in this case. Depending on the assignment of $\psi_{21}$, we can make the probability of having two cases (upper or lower circle) equal. Hence, in this scenario, the total number of bits 


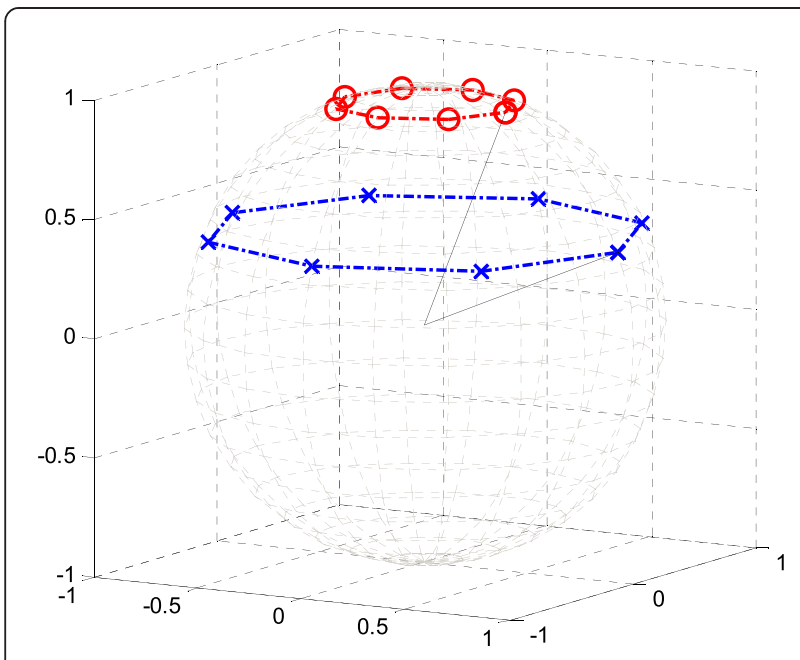

Figure 1 Distribution of quantized 2-by-1 beamforming vector based on fixed feedback rate.

representing $\mathbf{w}$ is $1+2=3$ or $1+4=5$, which is 4 bits on average (if the probability $\psi_{21}$ to be large and small, hence the probability of upper and lower circle are the same).

An optimal codebook obtained by VQ methodology as described in [5] is shown in Figure 3. The "optimum" codebook design criterion is to maximize the mean squared inner product (MSIP) of the beamforming vector and the codebook vectors. A modified form of the generalized Lloyd algorithm was used to train the codebooks used in the comparison. During the iterative training process, the nearest neighborhood condition was satisfied by identifying the partition cell $R_{i}$ corresponding to the $i$ th codebook vector that generates the highest MSIP with the training vector $\boldsymbol{v}$. At the end of

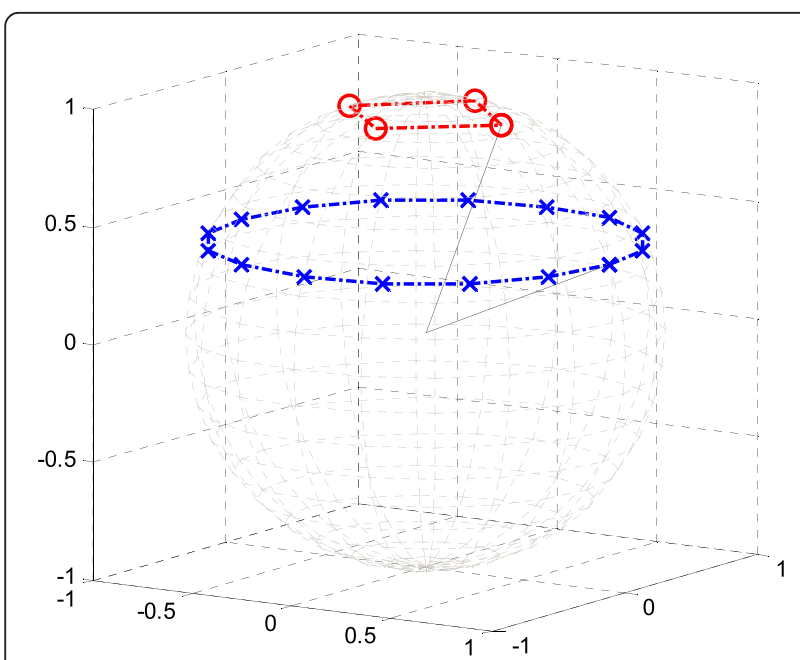

Figure 2 Distribution of quantized 2-by-1 beamforming vector based on variable feedback rate.

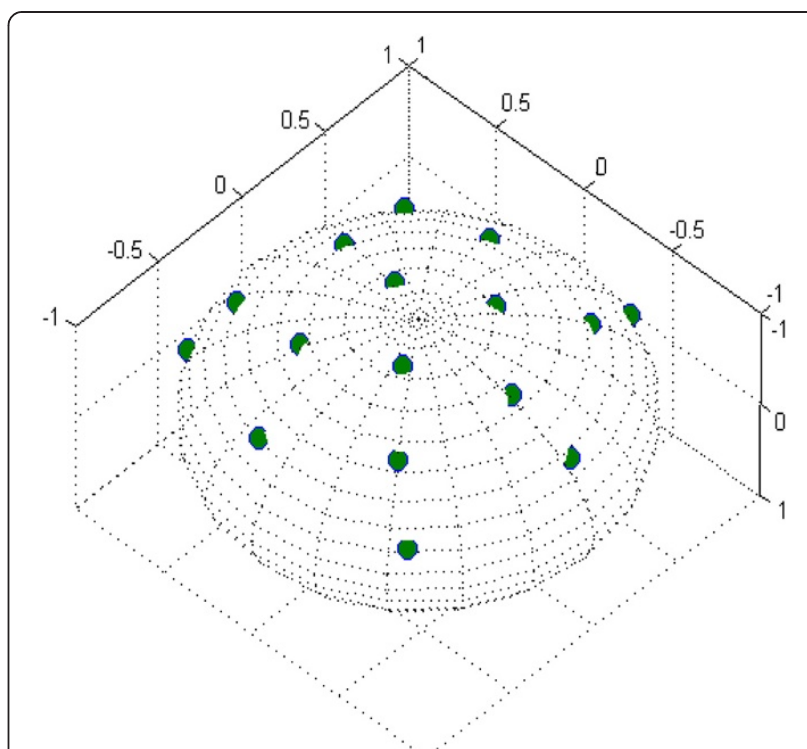

Figure 3 Optimal codebook trained using the maximum MSIP criterion.

an iteration, the principal eigenvector of $E\left[\boldsymbol{v} \boldsymbol{v}^{\mathrm{T}} \mid \boldsymbol{v} \in R_{i}\right]$ was computed and became the $i$ th codebook vector. From Figure 3, it can be seen that when using a variable feedback rate the codebook that appears in Figure 2 is closer to the optimal codebook than the one with a fixed feedback rate as shown in Figure 1.

\section{Efficient source coding}

In Figure $4 \mathrm{a}-\mathrm{f}$, we show the distribution of the GR parameters for a unitary beamforming matrix of dimension 3 $\times 2$ when $\mathrm{H}$ is Rayleigh i.i.d. fading channel. Through our studies of 5,000 channel matrices, we observe that the parameter $\phi$ exhibits a uniform distribution from $0^{\circ}$ to $360^{\circ}$ (i.e., 0 to $2 \pi$ ), while the parameter $\psi$ has a non-uniform distribution from the range of $0^{\circ}$ to $90^{\circ}$ (i.e., 0 to $\pi / 2$ ). We also observe an asymmetric distribution for $\psi_{31}$ and a symmetric distribution for $\psi_{21}$ and $\psi_{32}$. For channel matrices with other dimensions, we have similar observation.

The distributions of the quantized version of $\psi$ when using four levels of granularity are shown in Figure $4 \mathrm{~g}-\mathrm{i}$. So, we should use less bits to source code those values with higher occurring probability, and more bits to source code those values with lower occurring probability. One possibility is the use of Huffman source coding [14].

\section{Codebook design}

Due to the non-uniform and asymmetric distribution of some of the parameters, instead of quantizing the GR parameters uniformly, we can design a codebook so as to reduce the quantization error. For example, instead of quantizing $\psi_{31}$ uniformly with 2 bits using the value 
(a) $\phi_{11}$

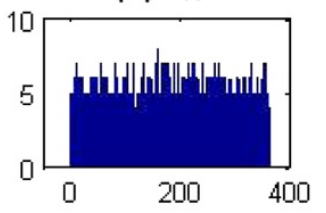

(d) $\psi_{21}$

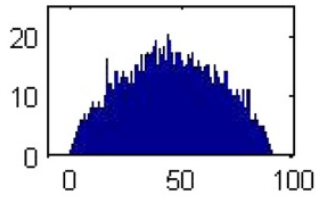

(g) 2 bits quanz of $\psi_{21}$

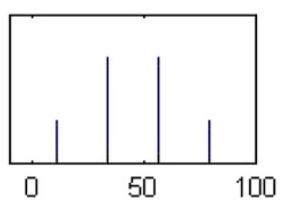

(b) $\phi_{21}$

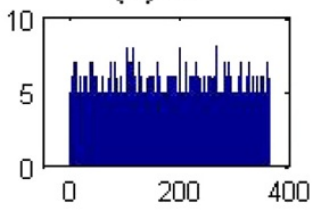

(e) $\psi_{31}$

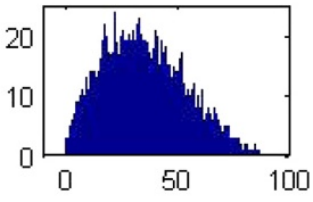

(h) 2bits quanz of $\psi_{31}$

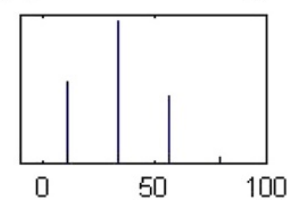

(c) $\phi_{22}$

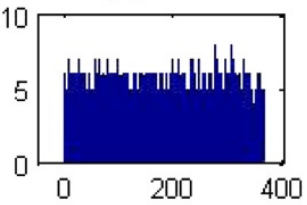

(f) $\psi_{32}$

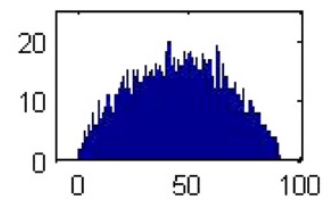

(i) 2 bits quanz of $\psi_{32}$

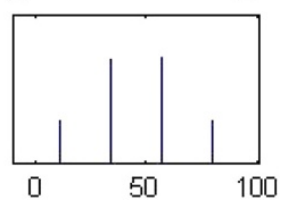

Figure 4 Distribution of the GR parameter for a $3 \times 2$ beamforming matrix.

$[11.25,33.75,56.25,78.75]$, we can use a codebook [8, $25,41,62]$ that is also 2 bits. As shown in Figure $4, \psi_{31}$ has a distribution that concentrate to the left-hand side (i.e., higher chances for smaller value), hence our codebook of $[8,25,41,62]$ also tends to have a lower value than the uniform codebook of $[11.25,33.75,56.25$, 78.75].

The above three techniques can be combined and optimized by certain design criteria, which can be a function of receiver. We will perform two case studies in the following sections, one based on the techniques $\mathrm{A}$ and $\mathrm{C}$, and another based on the techniques $\mathrm{A}$ and $\mathrm{B}$.

\section{Case studies}

Depending on the training symbol placement and the receiver design, we consider two cases. In the first case, a simple receiver is not retrained with the beamforming matrix, hence the receiver does not take the mismatch of the quantized beamforming matrix into account, and it simply uses a parallel decoder. On the other hand, in the second case, the receiver is retrained with the

Table 1 Number of bits allocation for three transmit antennas beamforming vector

\begin{tabular}{|c|c|c|c|c|c|c|c|c|c|}
\hline \multirow{3}{*}{$\begin{array}{l}\text { Average } \\
\text { feedback } \\
\text { bits }\end{array}$} & \multicolumn{9}{|c|}{ Bits allocated } \\
\hline & \multicolumn{4}{|c|}{ Givens } & \multicolumn{5}{|c|}{ Proposed } \\
\hline & $\mathbf{b}_{\Psi_{31}}$ & $\mathbf{b}_{\Psi_{21}}$ & $\mathbf{b}_{\varphi_{21}}$ & $\mathbf{b}_{\varphi_{11}}$ & $\mathbf{b}_{\Psi_{31}}$ & $\mathbf{b}_{\Psi_{21}}$ & $\mathbf{b}_{\varphi_{21}}$ & $\mathbf{b}_{\varphi_{11}}$ & Remark \\
\hline 8 & 1 & 1 & 3 & 3 & 1 & 1 & $2,3,4$ & $2,3,4$ & Table 2 \\
\hline 12 & 2 & 2 & 4 & 4 & 2 & 2 & $3,4,5,6$ & $3,4,5,6$ & Table 3 \\
\hline
\end{tabular}

updated beamforming matrix, hence the mismatch between the quantized beamforming matrix and the channel is taken into account. It uses a more complicated receiver, such as an MMSE receiver.

\section{Receiver with simple parallel decoder}

In this section, we consider the receiver as stated in (8), which is repeated below by taking into account the mismatch in the beamforming matrix with the channel

$$
\mathbf{a}=\overline{\mathbf{D}}^{-1} \mathbf{U}^{\mathrm{H}} \mathbf{y}=\overline{\mathbf{V}} \widetilde{\mathbf{W}} \mathbf{u}+\widetilde{\mathbf{n}}
$$

Due to quantization, $\mathbf{V} \widetilde{\mathbf{W}}$ is no longer an identity matrix, therefore such a simple receiver should be highly sensitive to the quantization error.

In this section, we demonstrate in details how the proposed scheme works for a $3 \times 1$ beamforming vector $\mathbf{w}$ as shown in (18). In Table 1, we show the bit assignment

Table 2 Bit allocation for $\varphi_{21}$ and $\varphi_{11}$ when the average number of feedback bit is 8

\begin{tabular}{|c|c|c|c|c|}
\hline \multicolumn{2}{|c|}{ Bit representative of } & \multicolumn{2}{|c|}{ Bits allocated for $\varphi_{21}$ and $\varphi_{11}$} & \multirow{2}{*}{$\begin{array}{l}\text { Total } \\
\text { number } \\
\text { of } \\
\text { feedback } \\
\text { bits }\end{array}$} \\
\hline$\psi_{31}^{*}$ & $\psi_{21}^{*}$ & $\mathbf{b}_{\varphi_{21}}$ & $\mathbf{b}_{\varphi_{11}}$ & \\
\hline 0 & 0 & 3 & 4 & 9 \\
\hline 0 & 1 & 4 & 3 & 9 \\
\hline 1 & 0 & 2 & 3 & 7 \\
\hline 1 & 1 & 3 & 2 & 7 \\
\hline
\end{tabular}

"Possible values for $\psi_{31}$ and $\psi_{21}$ are [17 50] degree. 
Table 3 Bit allocation for $\varphi_{21}$ and $\varphi_{11}$ when the average number of feedback bit is $\mathbf{1 2}$

\begin{tabular}{lllll}
\hline Bit representative of & \multicolumn{2}{l}{$\begin{array}{l}\text { Bits allocated } \\
\text { for } \boldsymbol{\varphi}_{\mathbf{2 1}} \text { and } \boldsymbol{\varphi}_{\mathbf{1 1}}\end{array}$} & $\begin{array}{l}\text { Total number of } \\
\text { feedback bits }\end{array}$ \\
\cline { 1 - 4 } $\boldsymbol{\psi}_{\mathbf{3 1}}^{*}$ & $\boldsymbol{\psi}_{\mathbf{2 1}}^{*}$ & $\mathbf{b}_{\boldsymbol{\varphi}_{\mathbf{2 1}}}$ & $\mathbf{b}_{\boldsymbol{\varphi}_{\mathbf{1 1}}}$ & \\
\hline 00 & 00 & 3 & 6 & 13 \\
00 & 01 & 4 & 5 & 13 \\
00 & 10 & 5 & 4 & 13 \\
00 & 11 & 6 & 3 & 13 \\
01 & 00 & 3 & 5 & 12 \\
01 & 01 & 4 & 4 & 12 \\
01 & 10 & 4 & 4 & 12 \\
01 & 11 & 5 & 3 & 12 \\
10 & 00 & 3 & 5 & 12 \\
10 & 01 & 4 & 4 & 12 \\
10 & 10 & 4 & 4 & 12 \\
10 & 11 & 5 & 3 & 12 \\
11 & 00 & 3 & 4 & 11 \\
11 & 01 & 3 & 4 & 11 \\
11 & 10 & 4 & 3 & 11 \\
11 & 11 & 4 & 3 & 11 \\
\hline
\end{tabular}

"Possible values for $\psi_{31}$ and $\psi_{21}$ are [8 2541 62] degree.

for the GR of the traditional and the proposed schemes. For example, in an average of 8 bits feedback configuration, the traditional scheme allocates 1 bit each to $\psi_{21}$ and $\psi_{31}$, and 3 bits each to $\phi_{11}$ and $\phi_{21}$. In the proposed scheme, 1 bit is assigned to $\psi_{21}$ and $\psi_{31}$, but 2,3 , or 4 bits to $\phi_{11}$, with the actual assignment (whether 2, 3, or 4 bits) depending on the value of $\psi_{21}$ and $\psi_{31}$, as shown in Table 2. For an average feedback rate of 12 bits, the actual bit assignment is shown in Table 3.

The quantization and reconstruction of $\phi$ are based on the formula in (13). For the $\psi$ parameter, since $\psi_{31}$ and $\psi_{21}$ are not uniformly distributed as shown in Figure 4, we design a codebook for those two parameters, the codebook used are shown as the footnote to Tables 2 and 3. Hence, in this case study, we have used the technique "dynamic bit assignment" and "codebook design" that have been mentioned in the previous section.

$$
\mathbf{w}=\left[\begin{array}{c}
r_{1} e^{j \phi_{11}} \\
r_{2} e^{j \phi_{21}} \\
r_{3}
\end{array}\right]=\left[\begin{array}{c}
\cos \psi_{21} \cos \psi_{31} e^{j \phi_{11}} \\
\sin \psi_{21} \cos \psi_{31} e^{j \phi_{21}} \\
\sin \psi_{31}
\end{array}\right]
$$

Since the receiver is not retrained with the beamforming matrix, the quantization error will be critical in this case. We first compare the error in quantization by using mean square error (MSE) in (19) or mean angular distance (MAD) [6] in (20).

$$
\begin{aligned}
& M S E=\mathrm{E}|\mathbf{w}-\widetilde{\mathbf{w}}|^{2} \\
& M A D=\mathrm{E} \sqrt{1-|\mathbf{w} \cdot \widetilde{\mathbf{w}}|^{2}}
\end{aligned}
$$

where $\bullet$ in (20) denotes the dot product operation.

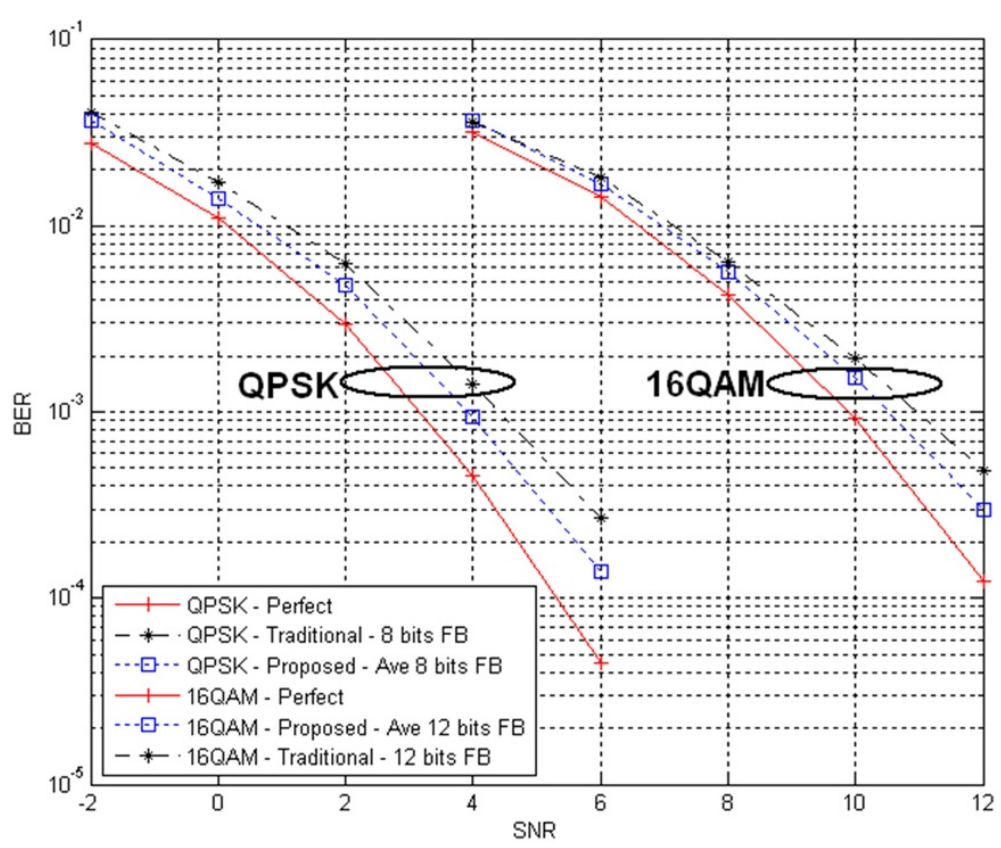

Figure 5 BER performance for 3 tx 3 rx, one stream based on SVD beamforming: QPSK with average 8 bits feedback and 16QAM with average 12 bits feedback. 
Table 4 Huffman code for GR parameters

\begin{tabular}{|c|c|c|c|c|}
\hline \multicolumn{5}{|l|}{ GR parameters $\psi_{21}$ and $\psi_{32}$} \\
\hline Quantized value of $\Psi_{21}$ or $\Psi_{32}$ & Probability (i) & Huffman code & Bits for $\Psi_{21}$ or $\Psi_{32}$ (ii) & Ave bits for $\Psi_{21}$ or $\Psi_{32}$ (i) $\times$ (ii) \\
\hline 11.25 & 0.14714 & 110 & 3 & 0.44142 \\
\hline 33.75 & 0.35496 & 0 & 1 & 0.35496 \\
\hline 56.25 & 0.35146 & 10 & 2 & 0.70292 \\
\hline \multirow[t]{2}{*}{78.75} & 0.14644 & 111 & 3 & 0.43932 \\
\hline & & & & 1.93862 \\
\hline \multicolumn{5}{|l|}{ GR parameters $\psi_{31}$} \\
\hline Quantized value of $\psi_{31}$ & Probability (i) & Huffman code & Bits for $\Psi_{31}$ (ii) & Ave bits for $\Psi_{31}$ (i) $\times$ (ii) \\
\hline 11.25 & 0.2722 & 10 & 2 & 0.5444 \\
\hline 33.75 & 0.47748 & 0 & 1 & 0.47748 \\
\hline 56.25 & 0.2299 & 110 & 3 & 0.6897 \\
\hline \multirow[t]{2}{*}{78.75} & 0.02042 & 111 & 3 & 0.06126 \\
\hline & & & & 1.77284 \\
\hline
\end{tabular}

MSE readings for quantization of $3 \times 1$ beamforming vector based on the traditional fixed rate feedback approach versus that of the newly proposed scheme based on variable rate feedback are as follows: MSE of 0.11 versus 0.091 (for 8 bits feedback) and MSE of 0.03 versus 0.028 (for 12 bits feedback). MAD readings are as follows: MAD of 0.31 versus 0.282 (for 8 bits feedback) and MAD of 0.162 versus 0.156 (for 12 bits feedback). Hence, the proposed scheme always achieves a lower MSE and MAD than the traditional scheme for both cases of average 8 or 12 bits feedback.

The BER performance is shown in Figure 5 for QPSK (with average 8 bits feedback) and 16QAM (with average 12 bits feedback) modulated $3 \times 3 \mathrm{MIMO}$, respectively. It can be seen that the proposed scheme outperforms the traditional approach. Notably such a performance gain is achieved without any additional feedback bandwidth.

\section{Receiver with MMSE detector}

In this section, we consider a different receiver from that in (8). It is assumed that the mismatch in the beamforming matrix is known, and we apply the MMSE detector as shown:

$$
\mathbf{a}=\left(\mathbf{G}^{\mathrm{H}} \mathbf{G}+\alpha \mathbf{I}\right)^{-1} \mathbf{G}^{\mathrm{H}} \mathbf{y} \quad \mathbf{G}=\mathbf{H} \widetilde{\mathbf{W}}
$$

In this case, we consider a three transmit antennas and two streams of data. Due to the non-uniform distribution of the parameters $\psi$ as shown in Figure 4, we can make use of Huffman coding [14] to encode the quantized value of $\psi$. Hence, we make use of the techniques "dynamic bit assignment" and "efficient source coding" discussed earlier in this case study.

As shown in Table 4, it is sufficient to represent the quantized version of $\psi_{21}$ (or $\psi_{32}$ ) and $\psi_{31}$ using 1.94 and 1.77 bits (instead of 2 bits for a granularity of four).

It is also found that when $\psi_{21}=33.75,56.25$, and $\psi_{31}=11.25,33.75$, the quantized version of the beamforming matrix will have higher chances of being poor in quality, hence we suggest that we use 3 bits to quantize $\phi_{11}$ and $\phi_{21}$ when the above-mentioned condition occurs. For the rest of the case, we simply use 2 bits to quantize $\phi_{11}$ and $\phi_{21}$. As shown in Table 5, it can be seen that 5.06 bits on average were required for the quantization of $\phi_{11}$ and $\phi_{21}$ in our proposed scheme.

Combining everything, the average number of bits required to represent a $3 \times 2$ unitary beamforming matrix using our proposed scheme can be computed as the following:

$$
\begin{gathered}
\text { Ave bits required: } \mathrm{E}\left[b_{\psi_{21}}\right]+\mathrm{E}\left[b_{\psi_{31}}\right]+\mathrm{E}\left[b_{\phi_{11}}+b_{\phi_{21}}\right]+\mathrm{E}\left[b_{\psi_{32}}\right]+\mathrm{E}\left[b_{\phi_{22}}\right] \\
=1.93862+1.77284+5.0592+1.93862+2 \mathrm{~b} \\
=12.7093
\end{gathered}
$$

We compare five different quantization schemes in Table 6. It can be seen that our proposed scheme

Table 5 Bits assignment for GR parameters $\varphi_{11}$ and $\varphi_{21}$

\begin{tabular}{lllll}
\hline Bits for $\varphi_{\mathbf{1 1}}$ (i) & Bits for $\varphi_{\mathbf{2 1}}$ (ii) & Condition & Probability (iii) & $\begin{array}{l}\text { Ave bits for } \varphi_{11} \text { and } \\
\varphi_{\mathbf{2 1}}(\mathbf{( i )}+(\mathrm{ii}))^{*}(\mathrm{iii})\end{array}$ \\
\hline 3 & 3 & $\psi_{21}=33.75,56.25$ & $(0.35496+0.35146) *$ & 3.1776 \\
& & $\psi_{31}=11.25,33.75$ & $(0.2722+0.47748)=0.5296$ & 1.8816 \\
& 2 & Otherwise & $1-0.5296=0.4704$ & 5.0592 \\
\hline
\end{tabular}


Table 6 Five schemes for comparisons

\begin{tabular}{lll}
\hline & Bit assignment & Ave number of feedback bits \\
\hline Scheme A & Perfect feedback $\infty$ \\
Scheme B & $b_{\psi}=1$ and $b_{\varphi}=312$ \\
Scheme C & $b_{\psi}=2$ and $b_{\varphi}=212$ \\
Scheme D & $b_{\psi}=2$ and $b_{\varphi}=315$ \\
Scheme E (proposed) Tables 4 and 5 & 12.71
\end{tabular}

(Scheme E with average 12.71 bits of feedback) achieve similar performance as Scheme D that requires 15 bits of feedback. Hence, we save about 2 bits of feedback, and this saving can be significant when there is a large number of subcarriers, especially in the future broadband system. Compare to Schemes B and C, we achieve a better performance with 0.71 additional bits in the average number of feedback bit.

The simulation results of the BER for the five schemes are shown in Figure 6. We assume three transmit antennas and three receive antennas with eigen beamforming and two data streams: one 64QAM and the other 16QAM, hence a $10 \mathrm{bits} / \mathrm{s} / \mathrm{Hz}$ spectral efficiency is achieved. It can be seen that Schemes $B$ and $C$ gave the worst BER, while Scheme A delivered the best performance in terms of BER. Scheme D performed better than Schemes $B$ and $C$ by using three more average feedback bits. Our proposed scheme can capture most of the gain that can be provided by Scheme D, but we require only 0.71 additional feedback bits compared to the additional 3 bits required by Scheme D.
The newly proposed scheme can be considered as a hybrid of the traditional GR approach and VQ-based approach, i.e., we have a code book for the GR parameters $\phi$ and $\psi$. However, the new scheme has a lower storage requirement than those based on VQ codebooks.

The assignment of the number of bits and the codebook design in these case studies are just for illustration, there could be other assignment methods that lead to better performance, and different receiver or different system design may lead to different design criteria.

\section{Conclusions}

In this article, a simple quantization scheme has been presented for the unit-norm beamforming vector or unitary beamforming matrix based on variable-rate feedback. The basic idea is to provide for higher resolution in the dense area and lower resolution in the sparse area. The idea can directly be applied to the existing GR approach allocating variable bits to the $\phi$ parameter according to the value of $\psi$. Due to the non-uniform distribution of the GR parameter $\psi$, the performance can be further improved if we incorporate into the system efficient source coding and codebook design for GR parameters. Results show that the proposed scheme can achieve a lower MSE and lower MAD. The BER performance of the close-loop MIMO system based on the proposed quantization scheme also outperforms that of existing schemes.

The proposed idea is not restricted to the use of eigen-beamformer or GR which have been used as the

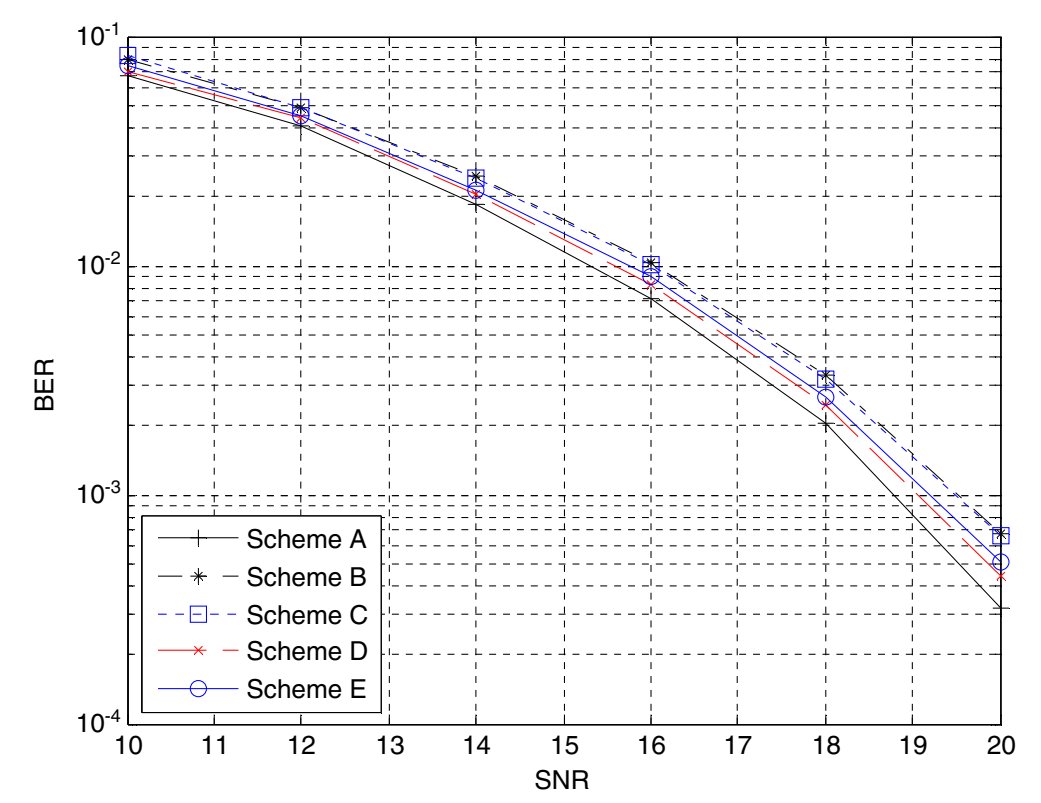

Figure 6 BER performance for 3tx 3rx two streams based on SVD beamforming with feedback bit assignment in Table 6 . First stream with 64QAM and second stream with 16QAM, total spectral efficiency $10 \mathrm{bps} / \mathrm{Hz}$. 
baseline for comparison. Our proposed method gives a better accuracy when compressing a unit-norm vector or unitary matrix, and such accuracy plays an important role in many communications system including precoding for multi-user MIMO.

\section{Competing interests}

The authors declare that they have no competing interests.

\section{Acknowledgment}

This study was partly supported by the Singapore University Technology and Design (grant no. SUTD-ZJU/RES/02/2011). Zhaoyang Zhang's was supported in part by the National Key Basic Research Program of China (No.

2012CB316104) and Zhejiang Provincial Natural Science Foundation of China (No. LR12F01002).

\section{Author details}

${ }^{1}$ Singapore University of Technology and Design, Singapore, Singapore. ${ }^{2}$ Institute for Infocomm Research, Singapore, Singapore. ${ }^{3}$ Cortex I.T. Labs, Melbourne, VIC, Australia. ${ }^{4}$ Zhejiang University, Hangzhou, China.

Received: 3 December 2011 Accepted: 5 June 2012

Published: 21 June 2012

\section{References}

1. IEEE P802.11n/D2.0, IEEE Standards Draft, February 2007

2. W.H. Chin, C. Yuen, Design of differential quantization for low bitrate channel state information feedback in MIMO-OFDM systems (Proc. IEEE VTC 2008 Spring, Singapore, 2008), pp. 827-831

3. M. Cho, W. Seo, Y. Kim, D. Hong, A joint feedback reduction scheme using delta modulation for dynamic channel allocation in OFDMA systems (Proc. of PIMRC, Berlin, 2005), pp. 2747-2750. vol. 4

4. IEEE P802.11ac/D0.2, IEEE Standards Draft, March 2011

5. J.C. Roh, B.D. Rao, Channel feedback quantization methods for MISO and MIMO systems, vol. 2 (IEEE PIMRC, Spain, 2004), pp. 805-809

6. D.J. Love, R.W. Heath, T. Strohmer, Grassmannian beamforming for multiple-input multiple-output wireless system. IEEE Trans. Inf. Theory 49, 2735-2747 (2003)

7. K.A. Chun, D.J. Love, On the performance of random vector quantization limited feedback beamforming in a MISO system. IEEE Trans. Wirel. Commun. 6, 458-462 (2007)

8. V. Raghavan, R.W. Heath, A.M. Sayeed, Systematic codebook designs for quantized beamforming in correlated MIMO channels. IEEE J. Sel. Ares Commun 25, 1298-1310 (2007)

9. S. Yang, J. Ko, Y. Lee, Transmit beamforming with reduced feedback information in OFDM based wireless systems (VTC Spring, Singapore, 2008), pp. 983-987

10. P. Wu, L. Li, P. Zhang, Unitary space vector quantization codebook design for precoding MIMO system. IEICE Trans. Commun. 91, 2917-2924 (2008)

11. J. Kim, C. Aldana, Efficient feedback of the channel information for closeloop beamforming in WLAN (IEEE VTC-Spring, Melbourne, 2006), pp. 2226-2230

12. J.C. Roh, B.D. Rao, Efficient feedback methods for MIMO channels based on parameterization. IEEE Trans. Wirel. Commun. 6(1), 282-292 (2007)

13. M.A. Sadrabadi, A.K. Khandani, F. Lahouti, Channel feedback quantization for high data rate MIMO systems. IEEE Trans. Wirel. Commun. 5, 3335-3338 (2006)

14. T.M. Cover, J.A. Thomas, Elements of Information Theory (Wiley Interscience, 1991)

doi:10.1186/1687-1499-2012-200

Cite this article as: Yuen et al:: Beamforming matrix quantization with variable feedback rate. EURASIP Journal on Wireless Communications and Networking 2012 2012:200.

\section{Submit your manuscript to a SpringerOpen ${ }^{\circ}$ journal and benefit from:}

- Convenient online submission

- Rigorous peer review

- Immediate publication on acceptance

- Open access: articles freely available online

- High visibility within the field

- Retaining the copyright to your article

Submit your next manuscript at $>$ springeropen.com 